\title{
Droplets Image Segmentation Method Based on Machine learning and Watershed
}

\author{
$\mathrm{He} \mathrm{Li}^{1}$, Shangshang Cheng ${ }^{1}$, Zhi Zhang ${ }^{2}$, Kaifei Zhang, ${ }^{1, *}$, Tahir Shahid Ali ${ }^{1}$ \\ ${ }^{1}$ School of Mechanical and Electrical Engineering, Henan Agricultural University, Zhengzhou 450002, \\ China \\ ${ }^{2}$ School of Agricultural Engineering, Jiangsu University, Zhenjiang 212013, China \\ *Corresponding Author.
}

\begin{abstract}
Watershed algorithm is used widely in segmentation of droplet overlapped spots on water-sensitive test paper. However, the phenomenon of over-segmentation, however, is often caused by noise and subtle changes of gray levels in images. To further improve segmentation accuracy of watershed algorithm, this paper proposes a cyclic iterative watershed segmentation algorithm. Through statistical analysis and logistic regression, machine learning models were classified to extract overlapping droplets on test papers. Loop iterative processing of seed points segments overlapping droplets with appropriate thresholds. Compared with fixed threshold watershed segmentation, this method has higher precision and efficiency for spray droplet evaluation in pesticide application.
\end{abstract}

Keywords: Image processing, overlapped droplets, segmentation, machine learning, watershed algorithm

\section{Introduction}

Pesticide spraying on crops is most common and effective application to prevent diseases, insects, pests and weeds. However excessive pesticide has a great impact on human health and environment ${ }^{[1]}$, advanced control and sensor technologies are applied for spray machinery to enhance pesticide utilization. Knowing the spray coverage is critically important for evaluating spraying process. Water-sensitive paper is used as artificial collectors to quantify spray application; Water-sensitive paper can provide an immediate assessment of spray coverage by making realtime decisions about performance of ongoing or planned spray applications in agricultural systems. Water-sensitive paper is overlapped with target areas or leaves and inspected after spraying. It was coated papers with a yellow surface that turns blue in the presence of aqueous droplets. However, there are some negative aspects, to analyze them manually or with the magnifying glass is difficult and time consuming during high humidity environments and with minute size droplets $\leq 50 \mu \mathrm{m}$. To quantify droplet deposition, many experts have done a lot of researches and developed several imaging systems to quickly obtain droplet coverage area on water-sensitive test paper. Zhu ${ }^{[2]}$ developed a portable scanning system and designed DepositsScan software that can quickly analyze distribution of droplets on water-sensitive paper. However, It needs to be improved. ${ }^{[3]}$ used image acquisition system to collect images by simulating actual spray situation in field, preprocessed images with enhancement and segmentation, and proposed an improved separation algorithm based on finding separation points between sticky droplets. ${ }^{[4]}$ designed a set of spray detection system with water-sensitive test paper based on computer vision acquisition software (Visual analogue scale, VAS), and achieved segmentation of sticky droplets by watershed segmentation but did not optimize over-segmentation problem of sticky. ${ }^{[5]}$ discriminated adhesive droplets based on shape factor and area threshold of droplet contour, counted adhesive droplets with limit etching method and iterative open operation algorithm, and called it watershed algorithm of iterative open operation labeling for segmentation. Segmentation of adhesive droplet images has a great influence on detection of deposition distribution characteristics. Existing systems mostly use watershed segmentation algorithms and corrosion segmentation algorithms to segment two or more adhesive droplet images. When using etching algorithm to segment the adhesive droplet, it is usually limited to large size of droplet and relatively light overlapped. Distance transform watershed segmentation method has a good effect on segmentation of strongly cohesive droplets, but it does not apply to situation that large droplets ISSN: 0010-8189

(C) CONVERTER 2020

www.converter-magazine.info 
adhere to small ones. Image processing algorithm of droplet on water-sensitive test paper was optimized and improved: Multiple droplet contours' characteristic parameters were used to construct a function for accurately identifying overlapped droplets, and a droplet recognition model was established based on machine learning technology. Watershed segmentation algorithm after distance transform is poor when large droplets overlap small droplets.

\section{Materials \& Methods}

\subsection{Droplet image acquisition}

Water-sensitive test paper is yellow in color, and water area quickly turns blue and forms droplet stains, which is easy with image processing. In this paper, the 3WQFTX-101S plant protection UAV produced by Quanfeng Aviation Plant Protection Company was selected for collection and test. The UAV was 2.0 meters away from water-sensitive paper, and spray operation was carried out at flight speeds of $3 \mathrm{~m} / \mathrm{s}, 4 \mathrm{~m} / \mathrm{s}$, and $5 \mathrm{~m} / \mathrm{s}$, respectively ${ }^{[6]}$. Paper obtained in test was collected through a smartphone and uploaded to a computer for image processing. Image processing program is based on open-source image project library OpenCV. It has a relatively complete image processing interface and can provide a rich function library. Analysis is convenient and accurate.

\subsection{Droplet image preprocessing}

To reduce computer operation, droplet image is preprocessed. Image preprocessing includes image de-noising and binary conversion. High-pass filtering method is used to de-noise overlapped droplet images, which is partial to subsequent extraction and segmentation of image. Dynamic threshold class segmentation method is used to perform automatic threshold processing to attain a binary image.

\subsection{Study on improved method of droplet overlapped judgment}

Image samples reveal that, phenomenon of overlapping is common and coverage density of detected droplets is low due to direct counting ${ }^{[7]}$. To accurately count, it is necessary to judge whether there is overlapped area in the image or not. If there is no overlapping, droplet parameters can be calculated directly; otherwise, extraction and segmentation will be carried out. This study is according to morphological characteristics of connected regions of droplet and some common features of discriminant fields of cells or circles, the following seven connected regions are determined

\subsubsection{Area}

For an $\mathrm{N} \times \mathrm{M}$ binary image, the area of region $\mathrm{R}$ can be expressed by scanning droplet image and calculating its total number of pixels with a pixel value of 1 .

\subsubsection{Length}

$$
\text { area }=\sum_{i=1}^{n} \sum_{j=1}^{n} f(i, j), \quad f(i, j)= \begin{cases}1, & (i, j) \in R \\ 0, & (i, j) \in R\end{cases}
$$

Length refers to total number of pixels of outline; it is usually measured by length of the polygon connecting midpoint of each pixel. If it is diagonal, it is root of 2-pixel grids, otherwise, it is 1-pixel grid length.

\subsubsection{Ratio}

It describes the degree of similarity between shape of object and circle, formula for calculating roundness of droplet image is:

$$
\text { ratio }=\frac{\text { area }}{\pi D_{\max ^{2}}}
$$

In formula (2) $D_{\max }$ - denotes maximum distance from the point on contour of droplet to centroid of the droplet, its unit is pixel

\subsubsection{Shape factor}

Image shape factor is a mathematical description of image features, which is often used to measure complexity of two-dimensional objects. Formula for calculating shape factor parameters of droplet image is:

ISSN: 0010-8189

(C) CONVERTER 2020

www.converter-magazine.info 


$$
f a c=\frac{4 \pi a r e a}{\text { length }^{2}}
$$

\subsubsection{Rectangle factor}

Rectangle factor is ratio of droplet contour area to minimum external rectangle area.

\subsubsection{Solidity}

Solidity is ratio of area of droplet contour to the area of the smallest convex polygon that contains the shape of object.

\subsubsection{Posture Ratio}

Posture ratio refers to aspect ratio of smallest circumscribed rectangle of droplet contour of object.

\section{Statistics and Analysis of Droplet Shape Parameters}

To explore the difference between different contour shape parameters 300 adhesive and single droplet images were selected for statistics and analysis of contour parameters. Fig. 1 shows partially selected images of the droplet. (a) single droplets (b) overlapped droplets.
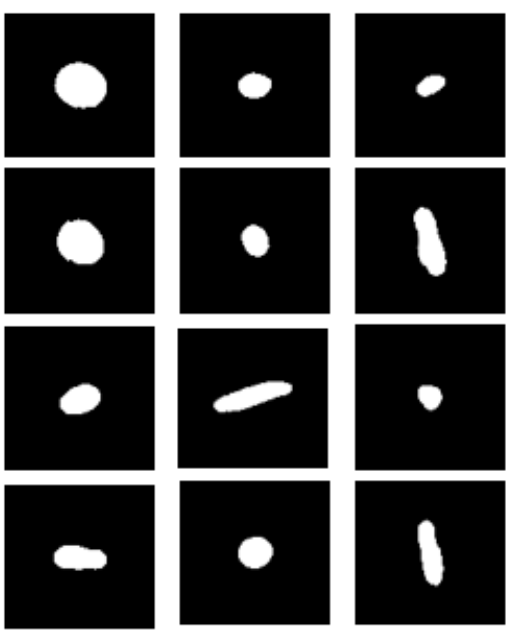

(a)

Figure 1: The binary image of selected droplet
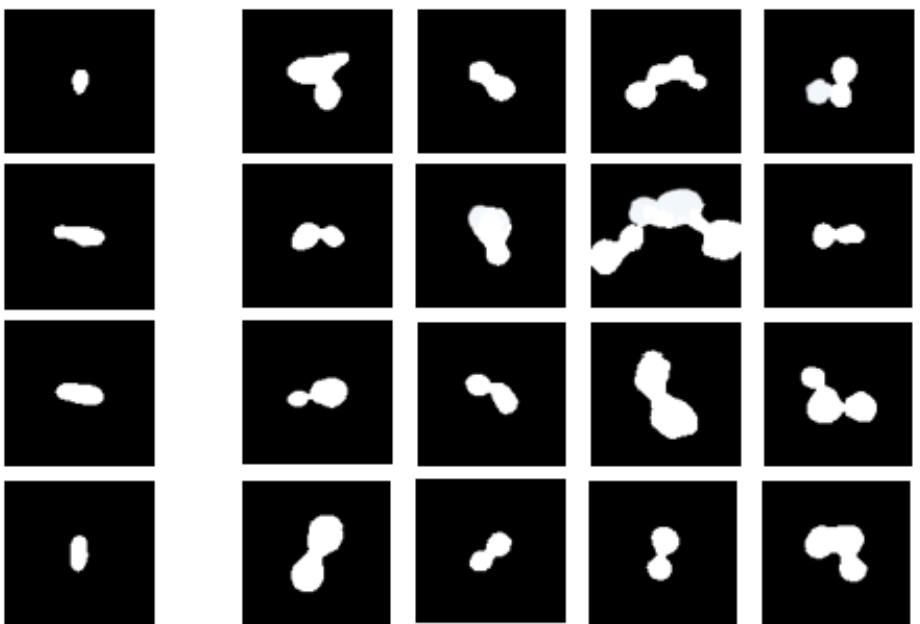

(b)

\subsection{Correlation analysis of droplet types}

Correlation analysis ${ }^{[8]}$ was conducted between shape parameters of different types of droplet contour and category of droplet (Table.1). Solidity parameters of droplet profile has highest correlation with droplet category $r=-0.80$. Although solid parameters of droplets have a great correlation with the type of droplets, most of them can be classified accurately. If solid parameters of droplet contour are taken as classification basis and 0.90 is taken as the evaluation standard, there are still many droplets that have not been successfully classified, and classification accuracy is still low.

Table 1 Correlation between shape parameters and droplet types

\begin{tabular}{|c|c|c|c|c|c|c|c|}
\hline parameter & area & length & ratio & Shape factor & Rectangle factor & solidity & Posture Ratio \\
\hline relevance & 0.48 & 0.60 & -0.42 & -0.68 & -0.72 & -0.80 & -0.08 \\
\hline
\end{tabular}

\subsubsection{Droplet type discrimination model}

Identification and screening of adhesive droplets can reduce workload of separation of adhesive droplets in the later stages, and can be used as an evaluation standard for accuracy of separation. Profile characteristic parameters of droplets containing area, length, ratio, shape factor, rectangle factor, solidity, and posture ratio were taken as input variables ${ }^{[9]}$. Different models were compiled to attain droplet type discrimination model i.e. machine

ISSN: 0010-8189

(C) CONVERTER 2020

www.converter-magazine.info 
learning models including K-nearest neighbor classification, logistic regression classification, decision tree classification and support vector machine (Fig. 2).

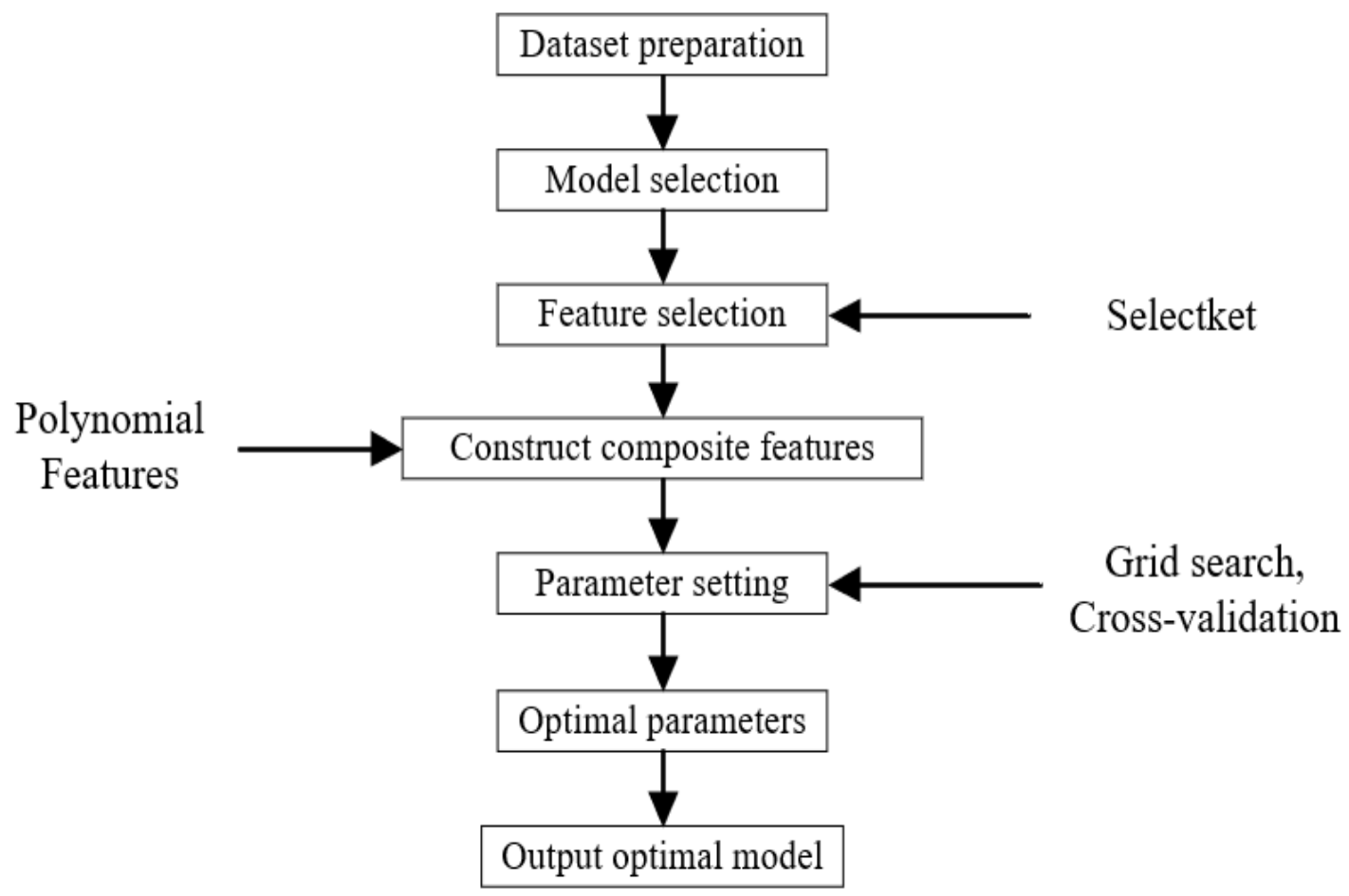

\subsection{Machine learning algorithms}

Figure 2: Classification model.

\subsubsection{K-nearest neighbors}

The k-Nearest Neighbors (k-NN) ${ }^{[10]}$ is simplest classification algorithms. k-NN is defined as a non-parametric lazy learning algorithm. However, algorithm needs all training dataset for classification process of a new instance. This results into costly processing time when the number of occurrences is very large. This method relies on determining $\mathrm{k}$ nearest neighbors among all the training dataset of new observation $\mathrm{x}$ by computing distances between $\mathrm{x}$ and each training data. Then, new instance gets the label (class) $\mathrm{y}$ of the predominant category among the $\mathrm{k}$ nearest neighbors. Choose value range of $\mathrm{k}$ [1 10], value range of Select Best parameter [1 7], Use Polynomial Features method to construct matching features, whose polynomial degree takes values $1,2,3,4$ in turn. The best parameters are obtained through grid search and cross-validation: Select Best takes a value of 2 , PolynomialFeatures polynomial degree takes a value of 3 , and $\mathrm{k}$ takes a value of 4 .

\subsubsection{Logistic Regressive}

Logistic regression is a method used for classification tasks with machine learning ${ }^{[11]}$. It belongs to "generalized linear model" and is mostly used for binary classification problems. The regular weight term C takes values 0.0001 , $0.001,0.01,0.1,1,10,100,1000$ in turn to build the model and regular term uses L1 norm and L2 norm to train separately. Use SelectBest to filter features, use PolynomialFeatures to build matching features, grid search and cross-validation result display that when the SelectKBest parameter takes the value 7, the polynomial degree of PolynomialFeatures is 3 , the regular term weight $\mathrm{C}$ is 10 , and the regular term is the $\mathrm{L} 1 \mathrm{norm}$, the model is optimal.

\subsubsection{Decision Tree}

Decision Tree is a class of machine learning nonlinear supervisory models with strong interpretability ${ }^{[12]}$. Feature selection criterion uses entropy and the Gini function. The maximum depth of decision tree is value [2-10]; minimum number of samples to be divided into internal nodes is a value [2-10]. Use SelectBest to filter features, and use PolynomialFeatures to build matching features. The grid search and cross-validation results show that the model is the best when the SelectKBest value is 3, the PolynomialFeatures polynomial degree is 3 , the maximum

ISSN: 0010-8189 
depth of the decision tree is 4 , the minimum number of samples required for subdividing internal nodes is 14 , and the information entropy is the excellent feature selection criterion.

\subsubsection{Support Vector Machine}

Support Vector Machine is a learning supervision model that minimizes structural risk. The penalty coefficient $\mathrm{C}$ values $0.001,0.001,0.01,0.1,1,10,100,100$.Kernel uses Gaussian function kernel; coefficient gamma values are $0.0001,0.001,0.01,0.1,1,10,100$. Use SelectBest to filter features, and use PolynomialFeatures to build matching features. The grid search and cross-validation results show that the SelectKBest parameter value is 3 , the polynomial degree of PolynomialFeatures is 3, the penalty coefficient $\mathrm{C}$ is 10 , and the kernel function coefficient gamma is 10 when the kernel function is constructed as the Gaussian kernel function. The model is optimal.

\subsubsection{Receiver operating characteristic curve}

Receiver operating characteristic curve is a comprehensive index of continuous variables of sensitivity and specificity, in evaluation of droplet classification model ${ }^{[13]}$, closer the curve is to upper left corner, higher the prediction accuracy of the model is. In the most extreme case, the curve is recombined with Y-axis, and prediction accuracy of model reaches $100 \%$. Area enclosed by the ROC curve and horizontal axis of model becomes prediction accuracy of model and is represented by AUC. Value range of AUC is [0,1]. Larger the value of AUC, higher becomes the prediction accuracy of model.

Figure 3 shows optimal parameters under different types of cloud droplets type classification model of ROC curve, curve of the logistic regression model is most close to upper left corner, followed by support vector machine classification model, decision tree classification model and the model of $\mathrm{K}$ nearest neighbor classification, four kinds of classification model in the prediction accuracy were $0.98,0.97,0.95,0.94$, and results show that: droplets by logistic regression model has highest type discriminant prediction accuracy.

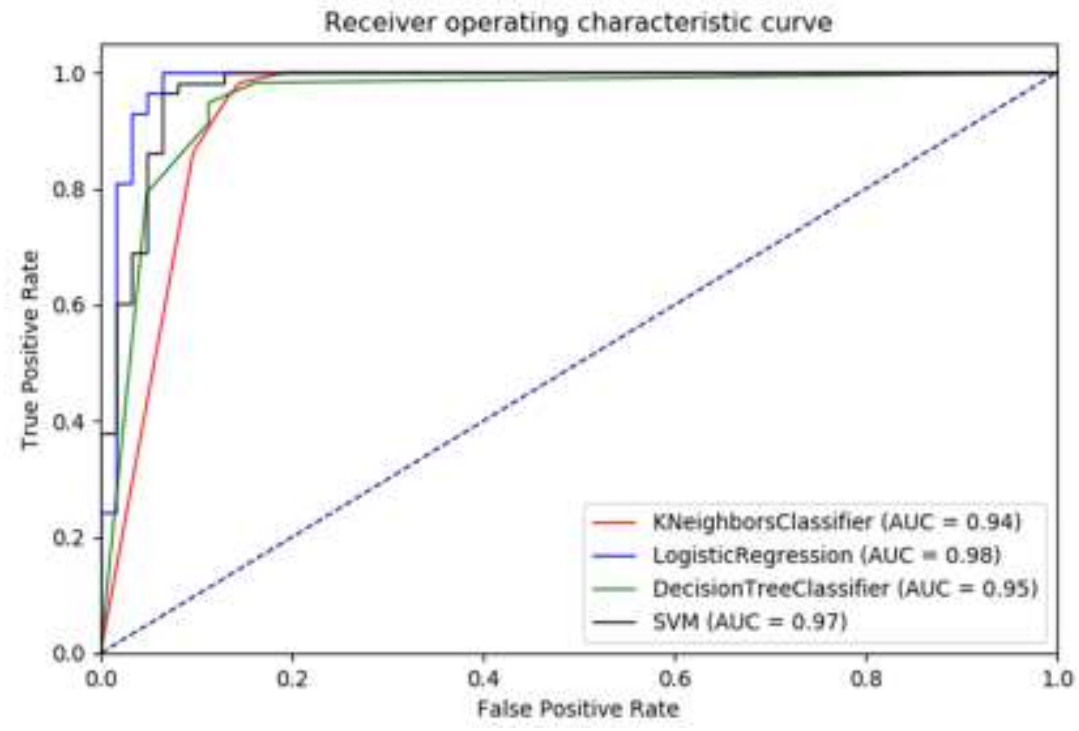

\section{Discussions}

Figure 3: ROC curve

\subsection{Method of image segmentation and rounding improvement for overlapped droplets}

In watershed segmentation algorithm based on topological theory, water-sensitive test paper image is regarded as a three-dimensional geological topological structure map, ${ }^{[14]}$ and gray value of each pixel in gradient image represents topographic height in geological topology map. Division of sticky droplets is realized in process of simulating water flow flooding the terrain by watershed. Fig. 4 and Fig. 5 respectively show three-dimensional geological topology of gradient image of water-sensitive test paper and binary image after distance transformation. Color in three-dimensional geological topology represents the size of gray value of each point.

ISSN: 0010-8189 


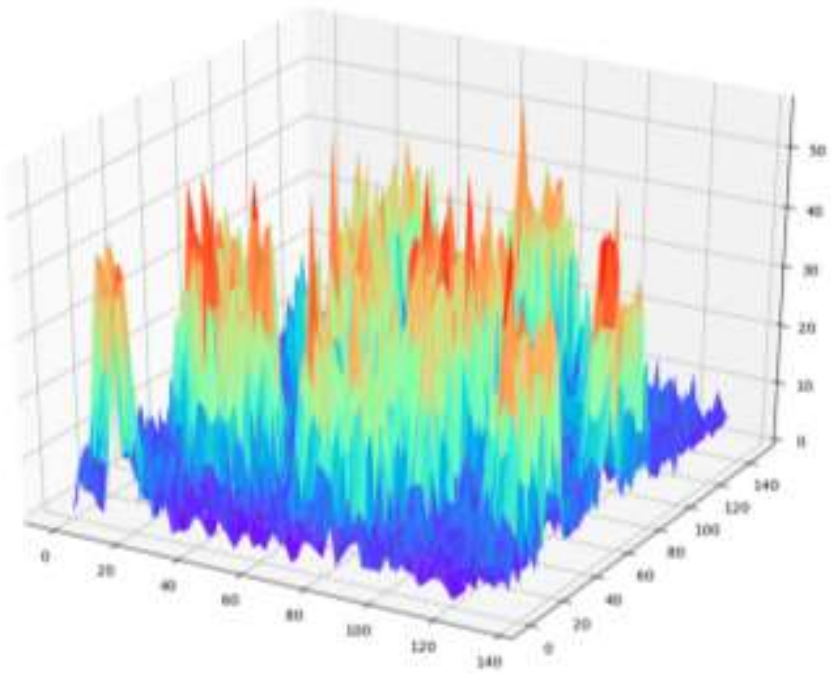

Figure 4: 3D view of original droplet image

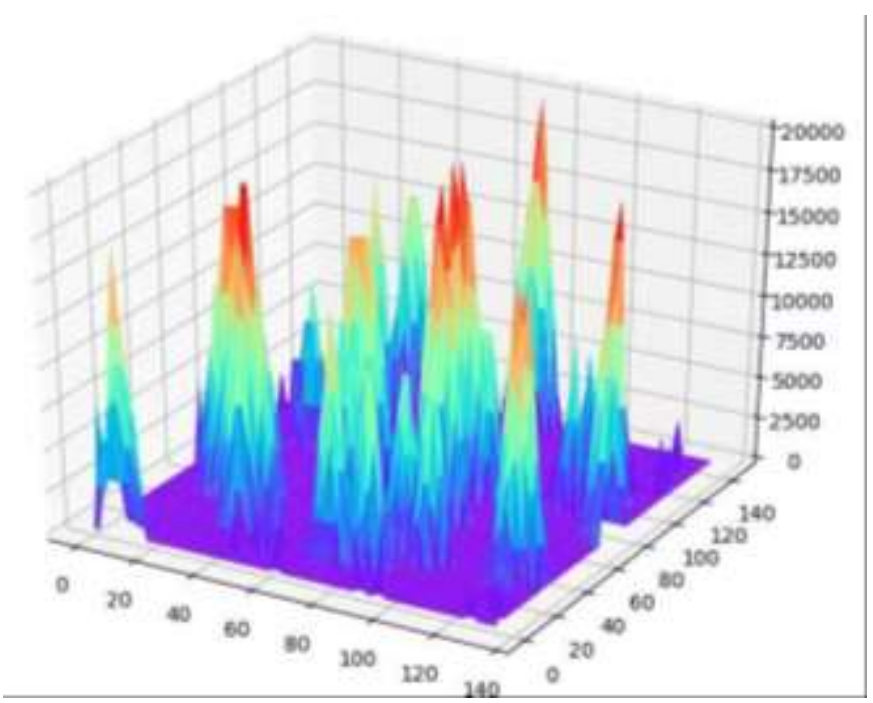

Figure 5: 3D view of original droplet image after distance transform

Segmentation of overlapped droplets can be realized by using watershed to simulate process of water flooding the terrain. However, when overlapped area of droplet is relatively large, there is no obvious change of gray level in overlapped area, segmentation effect of watershed segmentation algorithm is poor. Farther the pixel point in the droplet area is from the background, the higher the gray value of the point will be (Fig. 5). ${ }^{[15]}$. In the process of simulating water flow flooding, water will converge in area of sticky droplet, to realize segmentation of the sticky droplet. Therefore, watershed segmentation algorithm based on distance transformation is selected for segmentation of sticky droplets.

When it is used to separate adhesive objects, some small structural elements are usually generated due to interference of noise and other factors in image, which leads to phenomenon of over-segmentation. Choosing the same segmentation threshold for image segmentation will also lead to over-segmentation or under-segmentation. Therefore, based on established droplet recognition and classification model, a cyclic iterative watershed segmentation algorithm is proposed in this study.

As shown in Fig.6, droplets in water-sensitive test paper were identified and classified, both single and adhesive droplets were extracted, distance transform was carried out on image of the overlapped droplets, and results of distance transform were processed by morphological operation. Segmentation threshold $\theta=0.1$ was used to segment seed image, seed images were identified, then the single seed images were extracted, and the adhesive seed images

ISSN: 0010-8189

(C) CONVERTER 2020

Www.converter-magazine.info 
were segmented again with the threshold $\theta=0.1$, and repeated until the obtained seed images were all single seeds. The seed images obtained were summarized, and then watershed segmentation was performed on the images of the overlapped droplets. The segmented image of the overlapped droplets and image of non-overlapped droplets are combined to have resultant image. In this method, through cyclic processing of seed images, different adhesive droplet can be segmented with relatively appropriate segmentation thresholds, avoiding over-segmentation and inaccurate segmentation caused by overlapped segmentation with the same threshold for whole droplet image, as shown in Fig. 7. Image in segmentation process can be recognized, (a) is original binary image, (b) is single droplet image, (c) is overlapped droplet image,(d) is image of segmented seed points, (e) is image of segmented droplet.

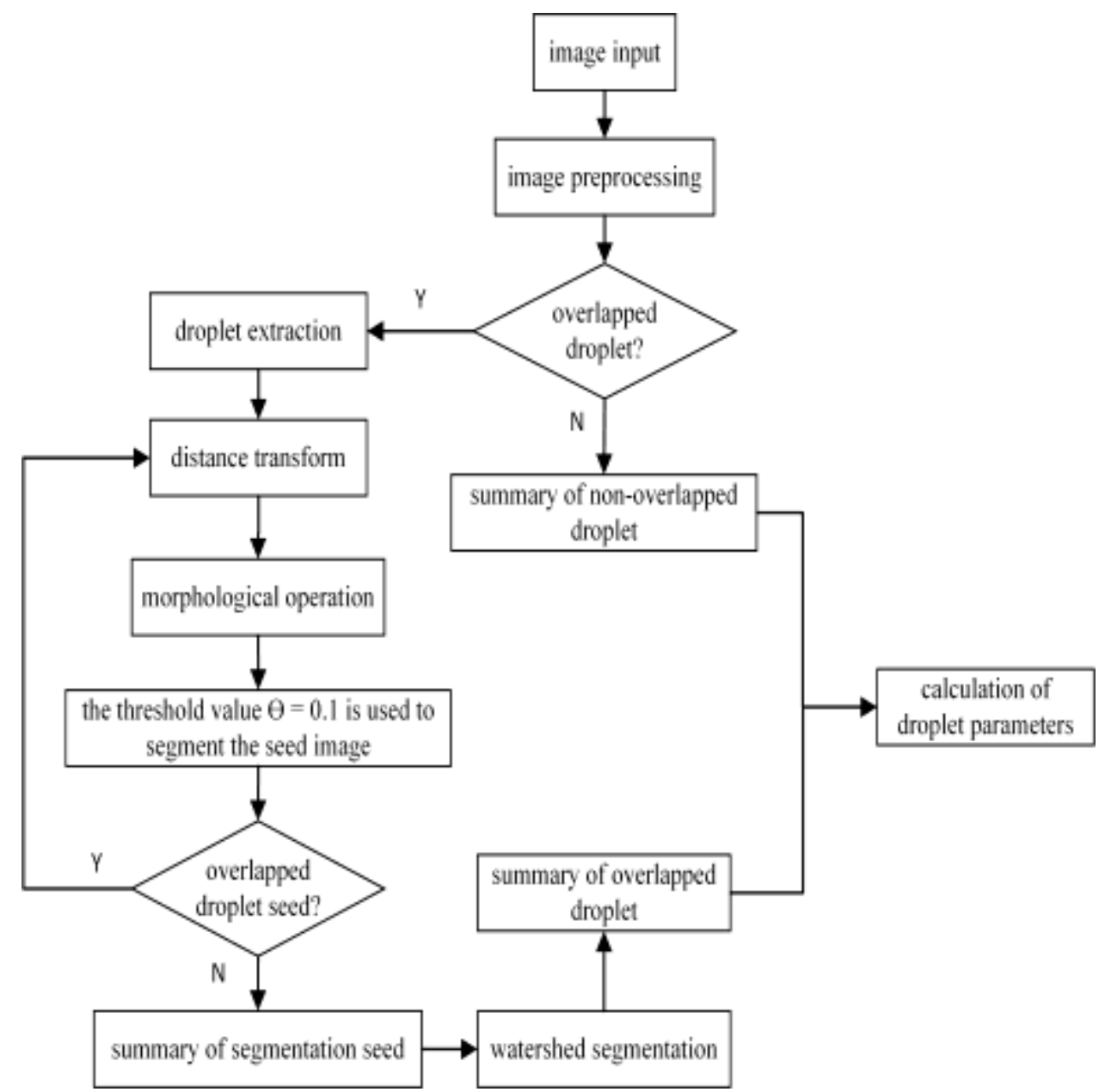

Figure 6: Segmentation algorithm

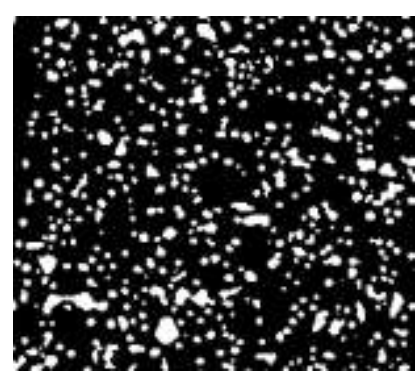

(a)

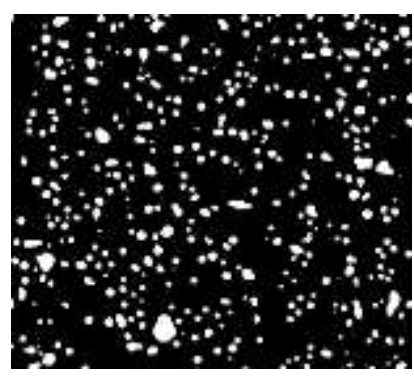

(b)

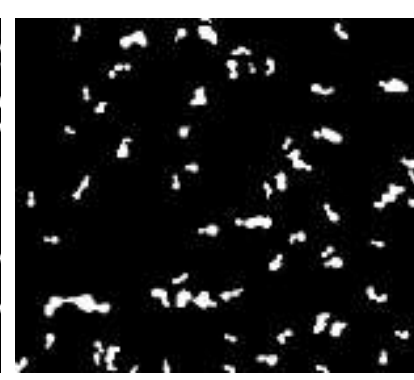

(c)

ISSN: 0010-8189 


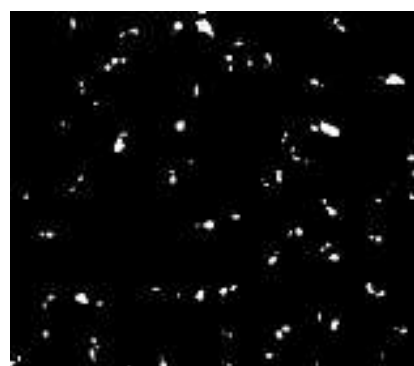

(d)

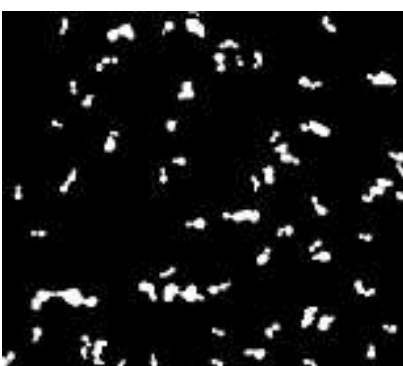

(e)

Figure 7: Segmentation process

In Figure 8(a-d) are ellipse figures with threshold from 0.2- 0.6. 8(a) shows minimization of adhesive droplet after overlapped segmentation. (b), (c), and (d) are ellipse fitting images with droplet overlapped after segmentation using fixed segmentation thresholds. Results obtained by using different segmentation thresholds are obviously different. The watershed segmentation with the segmentation threshold $\theta=0.2$ has a better effect. By comparing with the segmentation results above, it can be clearly seen that cyclic iterative watershed segmentation algorithm is superior to traditional watershed segmentation method for cohesive droplets.

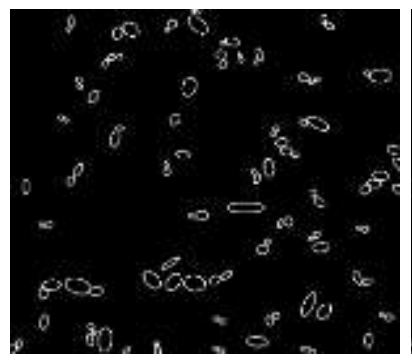

(a)

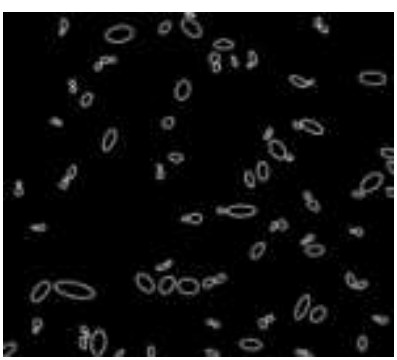

(b)

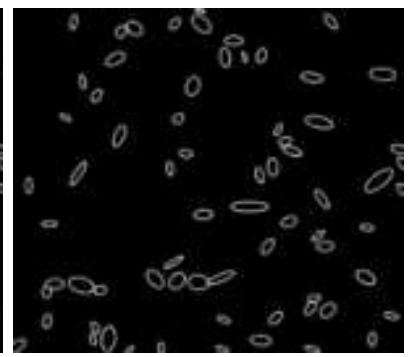

(c)

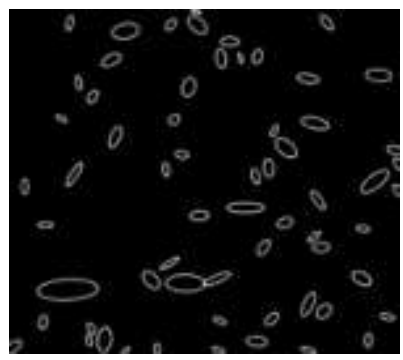

(d)

\section{Conclusion}

Figure 8: Ellipse fitting

This article analyzed the shape parameters of different types of droplets, and establishes a logistic regression classification model, under the optimal parameters, prediction accuracy of the model was 0.98 . We have designed a kind of loop iteration watershed segmentation algorithm, which is based on the seed image transformation of overlapped droplets, enables each overlapped droplet with an appropriate segmentation threshold. The segmentation results show that under-segmentation and over-segmentation are solved. It has great practical value for actual droplet statistical tasks during spraying and evaluation of various spraying techniques.

\section{Acknowledgements}

This work was financially supported by China Agriculture Research System (CARS-04-ps-25), the Science and Technology Project of Henan Province, China (212102110219).

\section{References}

[1] M. Cunha, Carvalho. C, Marcal. A, R. S, "Assessing the ability of image processing software to analyse spray quality on water-sensitive papers used as artificial targets". Biosystems Engineering, vol. 111, no.1, pp.11-23, 2012.

[2] H. Zhu, P. Salyani, M. Fox, R. D. "A portable scanning system for evaluation of spray deposit distribution". Computers and Electronics in Agriculture, vol. 76, no.1, pp.38-43, 2011.

[3] L. J. Qi, K. Hu, H. Wang, R. Ji, "Droplet Detection Based on Image Processing". Transactions of The Chinese Society of Agricultural Machinery, vol. 40, S1, pp. 48-51, .2009,

[4] Ozluoymak, O. B.; Bolat, A. "Development and assessment of a novel imaging software for optimizing ISSN: 0010-8189 
the spray parameters on water-sensitive papers". Computers and Electronics in Agriculture, vol. 168, 2020.

[5] Y. Wu, L. Qi, Y. Zhang; Z. Cheng, "Optimized Method of Improved Characteristics Judgment and Separation counting for Adhesive Droplets". Transactions of the Chinese Society of Agricultural Machinery, vol. 48, S1, pp. 220-227, 2017.

[6] G. Wang, Y. Han, X. Li, J. Andaloro, P. Chen, W. C. Hoffmann, Y. Lan, "Field evaluation of spray drift and environmental impact using an agricultural unmanned aerial vehicle (UAV) sprayer". Science of the Total Environment, vol. 737. pp.139793, 2020.

[7] A. Amaral, L.Rocha, O. Gonçalves, C. Ferreira, A. A.; Ferreira, E. C. "Application of image analysis to the prediction of EBC barley kernel weight distribution”, Industrial Crops and Products, vol. 30, no.3. pp.366-371, 2009.

[8] X. Wang, R. Lan, H. Wang, Z. Liu, X. Luo, "Fine-grained correlation analysis for medical image retrieval”. Computers \& Electrical Engineering, vol. 90. pp.106992, 2021.

[9] H. Fayed, A. Atiya, A. F. "Speed up grid-search for parameter selection of support vector machines". Applied Soft Computing, vol. 80, pp.202-210, 2019.

[10] K. Fredriksson, B. Braithwaite, "Quicker range- and k-NN joins in metric spaces. Information Systems”, vol. 52.pp.189-204, 2015.

[11] Van der Heijden, H. "Decision support for selecting optimal logistic regression models". Expert Systems with Applications, vol. 39, no.10, pp.8573-8583, 2012.

[12] Y. Yu, M. Shao, L. Jiang, Y. Ke, D. Wei, Zhanget al. "Quantitative analysis of multiple components based on support vector machine (SVM)”. Optik, vol. 237, pp.166759, 2021.

[13] L. Omar, I. Ivrissimtzis, "Using theoretical ROC curves for analysing machine learning binary classifiers”. Pattern Recognition Letters, vol. 128.pp.447-451, 2019.

[14] H. Li, C. Pan, Z. Chen, A. Wulamu, A. Yang, "Ore Image Segmentation Method Based on U-Net and Watershed”. Computers, Materials \& Continua, vol. 65, no.1, pp.563-578, 2020. 